\title{
A83 GENETIC MARKERS IN CLINICAL SUBTYPE OF JUVENILE IDIOPATHIC ARTHRITIS
}

P Le Blay, I Touitou, C Daïen, C Jorgensen, C Ludwig, M Rodière Unité médicale des maladies auto inflammatoires, Laboratoire de génétique, CHU Lapeyronie, Montpellier, France

10.1136/ard.2010.129619q

Background As the different subtypes of juvenile idiopathic arthritis (JIA) do not have the same severity and prognosis, they do not require the same therapeutic modalities. The goal is to obtain clinical remission with steroid-sparing strategy and identify prognosis markers.

Objective To identify genetic factors associated with clinical subtypes of JIA.

Methods The authors performed a monocentric retrospective study involving 70 children with JIA, classified into six subtypes by Edmonton's classification. The oligoarticular form was the most common. Severe JIA was defined by the polyarticular form, absence of therapeutic response, early onset and persistently elevated PCR. Thirteen single nucleotide polymorphisms (including PTPN 22, Stat 4, Traf 5, 1087-IL-10, 249 and 720-LTA, TGF $\beta 1,308$, 488 and 857-TNF $\alpha$; 196-TNf Rc2; C5-TRAF 1, TNFaIP3) were genotyped.

Results Genotype CC PTPN 22 was more strongly associated with the oligoarticular form ( $84 \%$ vs $16 \%$ in polyarticular form $(p=0.05))$. Other genes were less frequently found in the polyarticular form (such as Traf 5 and Stat 4), but did not reach statistical significance.

Conclusions Certain genotypes (including PTPN 22) seem to be associated with a lower risk of the polyarticular form of JIA. However, more patients are needed to validate these data. 